\title{
Starting with form, emerging with function: nanofibrous scaffolds for tissue engineering of
} orthopedic tissues
"This is an exciting time for the tissue engineering and regenerative medicine field as the complexity and usefulness of scaffolds, including nanofibrous scaffolds, continues to increase."

KEYWORDS: function $\approx$ nanofibrous scaffold $\approx$ orthopedics $\approx$ structure $\approx$ tissue engineering

\section{Structure \& function of orthopedic soft tissues}

The diverse set of movements that constitute our normal daily activities is orchestrated by the musculoskeletal system. Within individual joints, multiple soft tissues work in concert to control motion and transmit forces. To do so, the extracellular matrix of these materials is highly specialized to bear load in certain directions or patterns [1]. For example, in tissues that are designed to bear tensile load in one direction (e.g., tendons and ligaments), collagen fibers are assembled hierarchically into organized, rope-like structures. Without this efficient organization and assembly of component proteins, efficient mechanical function would not be achieved.

Unfortunately, musculoskeletal tissues are often injured during sports and work-related activities. Hundreds of thousands of ligament and tendon injuries occur each year in the USA alone, many of which require surgical intervention [2]. Meanwhile, resection of the knee meniscus due to injury or degeneration is the most commonly performed orthopedic surgery in the USA, with approximately 1 million operations each year [3]. Finally, lower back pain afflicts more than $20 \%$ of the general population, with degeneration of the soft tissues of the disc being a leading cause $[4,5]$. However, current surgical treatments often fail to restore the normal anatomy and structure of these tissues, at both the macro- and micro-scopic level, and, as a result, fail to restore long-term function $[6-8]$.

\section{Nanofibrous scaffolds for tissue engineering applications}

Tissue engineering and regenerative medicine approaches have made great progress in the last two decades in the generation of promising alternatives for the treatment of musculoskeletal injuries. Although many different techniques have been adopted to produce scaffold materials, the process known as electrospinning has rapidly gained popularity [1]. In this technique, a viscous polymer solution is drawn through a highly charged needle. When charge-charge repulsive forces within the solution overcome its intrinsic surface tension, a fine polymer jet is emitted, which travels rapidly to the nearest grounded surface. While in transit, these polymer fibers stretch and thin, such that their diameter reaches the nanoscale (typically a few hundred nanometers) upon deposition on a stationary collector. This fiber length scale matches the size typically observed for collagen fibrils in native tissues. Over time, scaffolds with random fiber organization are formed. Conversely, when the grounded collecting device rotated at high speeds, the fibers tend to align along the direction of rotation and scaffolds with aligned fibers can be generated.

Therefore, electrospinning provides a relatively simple method by which to form scaffolds that can recreate key microscopic features of the native tissue. However, orthopedic soft tissues typically feature fibrous organization at multiple length scales. As such, recent advances using nanofibrous scaffolds for tissue engineering of orthopedic tissues have focused on matching both the macroscopic and microscopic features of these complex tissues. In the following sections, we will briefly highlight a few of these recent innovations and conclude with a perspective on future directions and challenges still to be addressed.

Insertion sites of ligaments \& tendons Ligaments and tendons transmit tensile loads from bone to bone and muscle to bone,

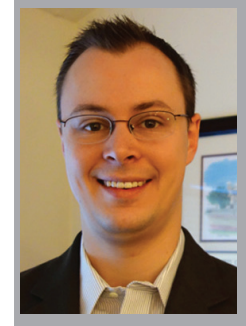

Matthew B Fisher

McKay Orthopedic Research Laboratory, Department of Orthopedic Surgery, Perelman School of Medicine, University of Pennsylvania. Philadelphia, PA 19104, USA and Philadelphia VA Medical Center, Philadelphia, PA 19104, USA

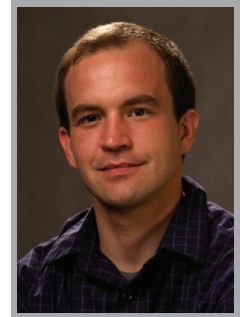

Robert L Mauck

Author for correspondence: McKay Orthopedic Research Laboratory, Department of Orthopedic Surgery, Perelman School of Medicine, University of Pennsylvania, 36th Street \& Hamilton Walk, Philadelphia, PA 19104, USA and Department of Bioengineering, School of Engineering \& Applied Science. University of Pennsylvania, Philadelphia, PA 19104, USA and Philadelphia VA Medical Center, Philadelphia, PA 19104, USA Tel.: +1 2158983294 Fax: +12155732133 lemauck@mail.med.upenn.edu 
respectively, to control joint motion. As such, they are dense tissues composed of uniformly aligned collagen fibers. The transitions from these tissues to bone or muscle are of considerable interest, as structural and mechanical features change in a gradual manner over a macroscopic scale (several millimeters) [9]. Recently, several groups have attempted to mimic this gradation in properties within a single electrospun scaffold. Ladd et al. created scaffolds utilizing two polymer sources with different mechanical properties [10]. These polymer sources were set a fixed distance apart, such that three distinct regions were created within the collecting fiber scaffolds: a soft polymer region; a mixed region; and a stiff polymer region. Tensile testing of these materials revealed a sixfold increase in modulus through these regions, and video strain analysis showed that the relative strain within the regions was similar to the native muscle-tendon junction. However, additional work is required for these scaffolds to match the mechanical properties of the native tissue, as muscle-tendon junctions can see relative increases in modulus of 500 -fold or more. Along similar lines, Li et al. developed a methodology to create scaffolds with gradated mineral content, as is observed in the native ligament to bone insertion [11]. These changes influenced both the local mechanical properties of the scaffolds as well as cellular interactions. These and other methods show great promise in the generation of nanofibrous scaffolds that can reproduce some of the complexity of native tissue interfaces.

\section{Meniscus of the knee joint}

Unlike ligaments and tendons, other tissues require organization along a more circuitous route. In the knee joint, the menisci help to distribute compressive loads and maintain joint congruency between the cartilage on the convex femoral condyles and the flat tibial plateau. Along the tibial plateau, the meniscus follows a general 'c-shaped' pattern with attachments to the plateau on either end. Between the femoral condyles and tibial plateau, the meniscus features a wedge-shaped cross-sectional geometry, which transforms pure compressive loads into a mixture of compressive axial and tensile circumferential loads. These latter loads are resisted by collagen fibers that traverse the tissue in a circumferential manner, consistent with its c-shape. In developing tissue-engineered scaffolds, this complex macroscopic geometry and microscopic organization must be mimicked to ensure proper function.
As an initial step we recently developed a novel approach to replicate the circumferential architecture of the meniscus within nanofibrous scaffolds [12]. To do so, we modified the collecting mandrel from a rotating cylinder, which collects aligned fibers in one direction, to a spinning planar disk. With this modification, we were able to generate scaffolds consisting of long nanofibers with a circumferential orientation. These scaffolds also had location-dependent mechanical properties, due to the changing fiber orientation. Moreover, cells seeded on these scaffolds were oriented along the direction of the fibers on the microscopic scale, but changed in a circumferential manner over the macroscopic scale. Therefore, this technique allows the collection of fibers with changing directionality within a single plane, and provides a critical step toward the formation of scaffolds that can match the complex fiber orientation of the knee meniscus.

\section{Annulus fibrosus of the intervertebral disc}

In other fibrous tissues, function is established across ordered planes, via the assembly of multilaminate structures. For instance, the intervertebral disc of the spine connects the bony vertebral bodies and consists of a gelatinous central nucleus pulposus and the surrounding fibrous annulus fibrosus (AF). The AF is composed of multiple layers, or lamella, featuring aligned collagen that alternates in directionality $\pm 30^{\circ}$ between adjacent lamella. The complex and hierarchical organization of the AF allows the intervertebral disc to resist the multidirectional forces and moments experienced about the spine.

\section{“...current surgical treatments often fail to restore the normal anatomy and structure of these tissues, at both the macro- and micro-scopic level, and, as a result, fail to restore long-term function."}

To mimic this complex hierarchical structure, Nerurkar et al. seeded individual aligned scaffolds with mesenchymal stem cells [13]. Following a preculture period, these layers were placed in apposition to each other at a $\pm 30^{\circ}$ angle and the composites were cultured in vitro for 10 weeks. Over time, the mesenchymal stem cells deposited a collagenous matrix with organization similar to the alignment of the scaffolds, and the constructs mimicked the multilamellar structure of native AF. Moreover, the 
interlamellar matrix produced by the cells had a positive effect on the overall tensile mechanics, due to the contribution of this material to shear resistance (i.e., prevention of fiber rotation). In a follow-up study, this interlamellar matrix also played a role under more complex (biaxial) loading configurations [14]. This lamellar reenforcing mechanism, predicated by nature, provides a novel means for reinforcing fibrous tissues in multiple directions, and illustrates how laboratory investigations can direct engineering approaches toward functional repair.

\section{Conclusion \& future perspective}

This is an exciting time for the tissue engineering and regenerative medicine field as the complexity and usefulness of scaffolds, including nanofibrous scaffolds, continues to increase [15]. Since first being employed in the early-to-mid 1990 s, the number of tissue engineering and regenerative medicine-related publications using electrospinning has accelerated. Since then, a wide variety of synthetic and natural polymers have been utilized [1]. Further work will include development of composite scaffolds produced from multiple polymers [16], increasing porosity $[17,18]$ and adding additional biologic functionality (through surface modification and drug delivery features) [19,20].

As highlighted above, work in the field of orthopedics is now addressing issues related to the formation of $3 \mathrm{D}$ structures with complex organization and hierarchy. As the size of these scaffolds increases to match the dimensions of the native tissue, a new slew of challenges will arise. First, the ability of cells to travel large distances (several millimeters), as well as diffusional limitations, will need to be addressed. Second, the mechanical properties of these 3D structures will need to be assessed in multiple directions and under the complex physiological loading conditions. Simulators and robotic testing systems can provide an initial assessment of scaffold performance within a particular joint, capturing its 'anatomic' mechanical functionality. Finally, rigorous preclinical evaluation of

\section{References}

1 Mauck RL, Baker BM, Nerurkar NL et al. Engineering on the straight and narrow: the mechanics of nanofibrous assemblies for fiber-reinforced tissue regeneration. Tissue Eng. Part B Rev. 15(2), 171-193 (2009).

2 United States Bone and Joint Decade. The Burden of Musculoskeletal Diseases in the these scaffolds will be required in large animal models.

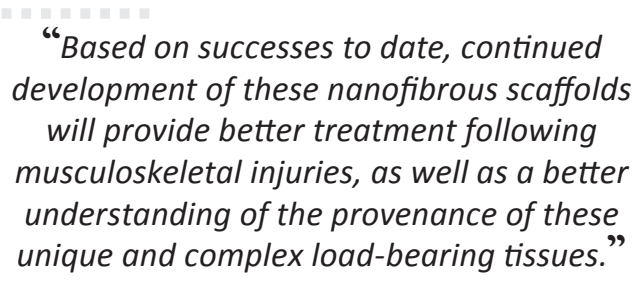

As scaffolds become more complex, other questions arise. One such question is how much of the native structure do nanofibrous scaffolds need to match in order to be effective? While it is well known that fiber alignment can induce cellular differentiation, as well as matrix production and orientation, it is not yet clear whether it is necessary to replicate every feature of the native tissue prior to implantation. It may well be that only a basic template is required from the outset (to instruct initial cell and matrix organization), with more complex interactions emerging naturally over time as the cells respond to their own particular loading environment. Based on successes to date, continued development of these nanofibrous scaffolds will provide better treatment following musculoskeletal injuries, as well as a better understanding of the provenance of these unique and complex load-bearing tissues.

\section{Financial \& competing interests disclosure}

The authors gratefully acknowledge funding from the NIH (R01EB02425 and R01AR056624), the Department of Veterans Affairs (I01RX000174), the Musculoskeletal Transplant Foundation (Junior Investigator Grant), the Penn Center for Musculoskeletal Disorders (P30AR050950) and the Penn Institute for Regenerative Medicine, which provided support for some of the work discussed. The authors have also filed several patents related to this work. The authors have no other relevant affiliations or financial involvement with any organization or entity with a financial interest in or financial conflict with the subject matter or materials discussed in the manuscript apart from those disclosed.

No writing assistance was utilized in the production of this manuscript.
United States. American Academy of Orthopaedic Surgeons, IL, USA (2008).

3 Garrett WE Jr, Swiontkowski MF, Weinstein JN et al. American Board of Orthopaedic Surgery Practice of the Orthopaedic Surgeon: part-II, certification examination case mix. J. Bone Joint Surg. Am. 88(3), 660-667 (2006).

4 Deyo RA, Mirza SK, Martin BI. Back pain prevalence and visit rates: estimates from U.S. national surveys, 2002. Spine (Phila. PA 1976) 31(23), 2724-2727 (2006).

5 Katz RT. Impairment and disability rating in low back pain. Clin. Occup. Environ. Med. 5(3), 719-740, viii (2006).

6 Woo SL-Y, Fisher MB, Feola AJ. Contribution of biomechanics to management of ligament and tendon injuries. Mol. Cell. Biomech. 5(1), 49-68 (2008). 
7 Fakouri B, Patel V, Bayley E, Srinivas S. Lumbar microdiscectomy versus sequesterectomy/free fragmentectomy: a long-term ( $>2 \mathrm{y})$ retrospective study of the clinical outcome. J. Spinal Disord. Tech. 24(1), 6-10 (2011).

8 Petty CA, Lubowitz JH. Does arthroscopic partial meniscectomy result in knee osteoarthritis? A systematic review with a minimum of 8 years' follow-up. Arthroscopy 27(3), 419-424 (2011).

9 Moffat KL, Sun WH, Pena PE et al. Characterization of the structure-function relationship at the ligament-to-bone interface. Proc. Natl Acad. Sci. USA 105(23), 7947-7952 (2008).

10 Ladd MR, Lee SJ, Stitzel JD, Atala A, Yoo JJ. Co-electrospun dual scaffolding system with potential for muscle-tendon junction tissue engineering. Biomaterials 32(6), 1549-1559 (2011).

11 Li X, Xie J, Lipner J, Yuan X, Thomopoulos $S$, Xia Y. Nanofiber scaffolds with gradations in mineral content for mimicking the tendon-to-bone insertion site. Nano Lett. 9(7), 2763-2768 (2009).

12 Fisher MB, Henning EA, Soegaard N, Esterhai JL, Mauck RL. Organized nanofibrous scaffolds that mimic the macroscopic and microscopic architecture of the knee meniscus. Acta Biomater. 9(1), 4496-4504 (2013).

13 Nerurkar NL, Baker BM, Sen S, Wible EE, Elliott DM, Mauck RL. Nanofibrous biologic laminates replicate the form and function of the annulus fibrosus. Nat. Mater. 8(12), 986-992 (2009).

14 Driscoll TP, Nakasone RH, Szczesny SE, Elliott DM, Mauck RL. Biaxial mechanics and inter-lamellar shearing of stem-cell seeded electrospun angle-ply laminates for annulus fibrosus tissue engineering. J. Orthop. Res. doi:10.1002/jor.22312 (2013) (Epub ahead of print).

15 Fisher MB, Mauck RL. Tissue engineering and regenerative medicine: recent innovations and the transition to translation. Tissue Eng. Part B Rev. 19(1), 1-13 (2013).
16 Baker BM, Nerurkar NL, Burdick JA, Elliott DM, Mauck RL. Fabrication and modeling of dynamic multipolymer nanofibrous scaffolds. J. Biomech. Eng. 131(10), 101012 (2009).

17 Baker BM, Shah RP, Silverstein AM, Esterhai JL, Burdick JA, Mauck RL. Sacrificial nanofibrous composites provide instruction without impediment and enable functional tissue formation. Proc. Natl Acad. Sci. USA 109(35), 14176-14181 (2012).

18 Zhong S, Zhang Y, Lim CT. Fabrication of large pores in electrospun nanofibrous scaffolds for cellular infiltration: a review. Tissue Eng. Part B Rev. 18(2), 77-87 (2012).

19 Ionescu LC, Lee GC, Sennett BJ, Burdick JA, Mauck RL. An anisotropic nanofiber/ microsphere composite with controlled release of biomolecules for fibrous tissue engineering. Biomaterials 31(14), 4113-4120 (2010).

20 Richardson TP, Peters MC, Ennett AB, Mooney DJ. Polymeric system for dual growth factor delivery. Nat. Biotechnol. 19(11), 1029-1034 (2001). 